\title{
Differential sandwich theorem for certain class of analytic functions associated with an integral operator
}

\author{
Luminiţa-Ioana Cotîrlă and Adriana Cătaş
}

\begin{abstract}
In this paper we obtain some applications of first order differential subordination and superordination result involving an integral operator for certain normalized analytic function.
\end{abstract}

Mathematics Subject Classification (2010): 30C45.

Keywords: Integral operator, subordination and superordination, analytic functions, sandwich theorem.

\section{Introduction and preliminaries}

Let $\mathcal{A}$ denote the class of functions of the form

$$
f(z)=z+\sum_{k=2}^{\infty} a_{k} z^{k}, a_{k} \geq 0
$$

which are analytic and univalent in the open unit disk $U=\{z:|z|<1\}$.

If $f$ and $g$ are analytic functions in $U$, we say that $f$ is subordinate to $g$ in $U$, written symbolically as $f \prec g$ or $f(z) \prec g(z)$ if there exists a Schwarz function $w(z)$ analytic in $U$, with $w(0)=0$ and $|w(z)|<1$, such that $f(z)=g(w(z)), z \in U$. In particular, if the function $g$ is univalent in $U$, the subordination $f \prec g$ is equivalent to $f(0)=g(0)$ and $f(U) \subset g(U)$ (see [2], [3]).

For the function $f$ given by (1.1) and $g \in \mathcal{A}$ given by $g(z)=z+\sum_{k=2}^{\infty} b_{k} z^{k}$, the Hadamard product (or convolution) of $f$ and $g$ is defined by

$$
(f * g)(z)=z+\sum_{k=2}^{\infty} a_{k} b_{k} z^{k}=(g * f)(z) .
$$


The set of all functions $f$ that are analytic and injective on $\bar{U}-E(f)$, denote by $Q$ where

$$
E(f)=\left\{\zeta \in \partial U: \lim _{z \rightarrow \zeta} f(z)=\infty\right\}
$$

and are such that $f^{\prime}(\zeta) \neq 0$ for $\zeta \in \partial U \backslash E(f)$, (see [4]).

If $\psi: \mathbb{C}^{3} \times U \rightarrow \mathbb{C}$ and $h$ is univalent in $U$ with $q \in Q$. In [3] Miller and Mocanu consider the problem of determining conditions on admissible functions $\psi$ such that

$$
\psi\left(p(z), z p^{\prime}(z), z^{2} p^{\prime \prime}(z) ; z\right) \prec h(z)
$$

implies that $p(z) \prec q(z)$ for all functions $p \in \mathcal{H}[a, n]$ that satisfy the differential subordination (1.2).

Let $\phi: \mathbb{C}^{3} \times U \rightarrow \mathbb{C}$ and $h \in \mathcal{H}$ with $q \in \mathcal{H}[a, n]$. In [4] and [5] is studied the dual problem and determined conditions on $\phi$ such that

$$
h(z) \prec \phi\left(p(z), z p^{\prime}(z), z^{2} p^{\prime \prime}(z) ; z\right)
$$

implies $q(z) \prec p(z)$ for all functions $p \in Q$ that satisfy the above subordination. They also found conditions so that the functions $q$ is the largest function with this property, called the best subordinant of the subordination (1.3).

Let $\mathcal{H}(U)$ be the class of analytic functions in the open unit disc.

For $n$ a positive integer and $a \in \mathbb{C}$ let

$$
\mathcal{H}[a, n]=\left\{f \in \mathcal{H}: f(z)=a+a_{n} z^{n}+\ldots\right\} .
$$

The integral operator $I^{m}$ of a function $f$ is defined in [6] by

$$
\begin{gathered}
I^{0} f(z)=f(z), \\
I^{1} f(z)=I f(z)=\int_{0}^{z} f(t) t^{-1} d t, \\
\ldots \\
I^{m} f(z)=I\left(I^{m-1} f(z)\right), z \in U .
\end{gathered}
$$

Lemma 1.1. [3] Let $q$ be univalent in $U, \zeta \in \mathbb{C}^{*}$ and suppose that

$$
\operatorname{Re}\left\{1+\frac{z q^{\prime \prime}(z)}{q^{\prime}(z)}\right\}>\max \left\{0,-\operatorname{Re}\left(\frac{1}{\zeta}\right)\right\} \text {. }
$$

If $p$ is analytic in $U$ with $p(0)=q(0)$ and

$$
p(z)+\zeta z p^{\prime}(z) \prec q(z)+\zeta z q^{\prime}(z)
$$

then $p(z) \prec q(z)$ and $q$ is the best dominant.

Lemma 1.2. [3] Let the function $q$ be univalent in the unit disk and let $\theta, \varphi$ be analytic in domain $D$ containing $q(U)$ with $\varphi(w) \neq 0$, where $w \in q(U)$. Set

$$
Q(z)=z q^{\prime}(z) \varphi(q(z)) \text { and } h(z)=\theta(q(z))+Q(z) .
$$

Suppose that

$$
\begin{gathered}
Q \text { is starlike univalent in } U ; \\
\operatorname{Re}\left\{\frac{z h^{\prime}(z)}{Q(z)}\right\}>0, \text { for } z \in U .
\end{gathered}
$$


If $p$ is analytic with $p(0)=q(0), p(U) \subseteq D$ and

$$
\theta(p(z))+z p^{\prime}(z) \varphi(p(z)) \prec \theta(q(z))+z q^{\prime}(z) \varphi(q(z))
$$

then $p(z) \prec q(z)$ and $q$ is the best dominant.

Lemma 1.3. [1] Let $q$ be convex in the unit disc $U, q(0)=a$ and $\zeta \in \mathbb{C}, \operatorname{Re}(\zeta)>0$. If $p \in \mathcal{H}[a, 1] \cap Q$ and $p(z)+\zeta z p^{\prime}(z)$ is univalent in $U$ then

$$
q(z)+\zeta z q^{\prime}(z) \prec p(z)+\zeta z p^{\prime}(z)
$$

implies $q(z) \prec p(z)$ and $q$ is the best subordinant.

Lemma 1.4. [2] Let the function $q$ be convex and univalent in the unit disc $U$ and $\theta$ and $\varphi$ be analytic in a domain $D$ containing $q(U)$. Suppose that

1. $\operatorname{Re}\left\{\frac{\theta^{\prime}(q(z))}{\varphi(q(z))}\right\}>0$ for $z \in U$ and

2. $Q(z)=z q^{\prime}(z) \varphi(q(z))$ is starlike univalent in $U$.

If $p \in \mathcal{H}[q(0), 1] \cap Q$ with $p(U) \subseteq D$ and $\theta(p(z))+z p^{\prime}(z) \varphi(p(z))$ is univalent in $U$ and

$$
\theta(q(z))+z q^{\prime}(z) \varphi(q(z)) \prec \theta(p(z))+z p^{\prime}(z) \varphi(p(z))
$$

then $q(z) \prec p(z)$ and $q$ is the best subordinant.

\section{Main results}

Theorem 2.1. Let $q$ be univalent in $U$, with $q(0)=1$ and $q(z) \neq 0$ for all $z \in U$, and let $\sigma \in \mathbb{C}^{*}, f \in \mathcal{A}$ and suppose that $f$ and $g$ satisfy the next conditions:

$$
\frac{I^{m+1}(f(z))}{z} \neq 0, z \in U
$$

and

$$
\operatorname{Re}\left\{1+\frac{z q^{\prime \prime}(z)}{q^{\prime}(z)}-\frac{z q^{\prime}(z)}{q(z)}\right\}>0 \text { for } z \in U \text {. }
$$

If

$$
\frac{I^{m}(f(z))}{I^{m+1}(f(z))} \prec 1+\frac{z q^{\prime}(z)}{\sigma q(z)},
$$

then

$$
\left(\frac{I^{m+1}(f(z))}{z}\right)^{\sigma} \prec q(z)
$$

and $q$ is the best dominant of (2.3).

Proof. Let

$$
p(z)=\left(\frac{I^{m+1}(f(z))}{z}\right)^{\sigma}, z \in U .
$$

Because the integral operator $I^{m}$ satisfies the identity $z\left[I^{m+1}(f(z))\right]^{\prime}=I^{m}(f(z))$ and the function $p(z)$ is analytic in $U$, by differentiating (2.4) logarithmically with respect to $z$, we obtain

$$
\frac{z p^{\prime}(z)}{p(z)}=\sigma\left(\frac{I^{m}(f(z))}{I^{m+1}(f(z))}-1\right) .
$$


In order to prove our result we will use Lemma 1.2. In this lemma we consider

$$
\theta(w)=1 \text { and } \varphi(w)=\frac{1}{\sigma w},
$$

then $\theta$ is analytic in $\mathbb{C}$ and $\varphi(w) \neq 0$ is analytic in $\mathbb{C}^{*}$. Also, if we let

$$
Q(z)=z q^{\prime}(z) \varphi(q(z))=\frac{z q^{\prime}(z)}{\sigma q(z)}
$$

and

$$
h(z)=\theta(q(z))+Q(z)=1+\frac{z q^{\prime}(z)}{\gamma \sigma q(z)}
$$

from (2.2) we see that $Q(z)$ is a starlike function in $U$. We also have

$$
\operatorname{Re}\left\{\frac{z h^{\prime}(z)}{Q(z)}\right\}=\operatorname{Re}\left\{1+\frac{z q^{\prime \prime}(z)}{q^{\prime}(z)}-\frac{z q^{\prime}(z)}{q(z)}\right\}>0 \text { for } z \in U
$$

and then, by using Lemma 1.2 we deduce that subordination $(2.3)$ implies $p(z) \prec q(z)$ and the function $q$ is the best dominant of (2.3).

Taking $q(z)=\frac{1+A z}{1+B z}(-1 \leq B<A \leq 1)$ in Theorem 2.1 , it easy to check that the assumption

$$
p(z)+\frac{1}{\sigma} z p^{\prime}(z) \prec q(z)+\frac{\alpha}{\sigma} z q^{\prime}(z)
$$

holds, hence we obtain the next result.

Corollary 2.2. Let $\sigma \in \mathbb{C}^{*}$ and $f \in \mathcal{A}$. Suppose

$$
\frac{I^{m+1}(f(z))}{z} \neq 0, z \in U
$$

If

$$
\frac{I^{m}(f(z))}{I^{m+1}(f(z))} \prec 1+\frac{z(A-B)}{\sigma(1+A z)(1+B z)}
$$

then

$$
\left(\frac{I^{m+1}(f(z))}{z}\right)^{\sigma} \prec \frac{1+A z}{1+B z}
$$

and $q(z)=\frac{1+A z}{1+B z}$ is the best dominant.

Taking $q(z)=\frac{1+z}{1-z}$ in Theorem 2.1, it easy to check that the assumption

$$
p(z)+\frac{1}{\sigma} z p^{\prime}(z) \prec q(z)+\frac{\alpha}{\sigma} z q^{\prime}(z)
$$

holds, hence we obtain the next result.

Corollary 2.3. Let $\sigma \in \mathbb{C}^{*}$ and $f \in \mathcal{A}$. Suppose

$$
\frac{I^{m+1}(f(z))}{z} \neq 0, z \in U
$$

If

$$
\frac{I^{m}(f(z))}{I^{m+1}(f(z))} \prec 1+\frac{2 z}{\sigma(1-z)(1+z)}
$$


then

$$
\left(\frac{I^{m+1}(f(z))}{z}\right)^{\sigma} \prec \frac{1+z}{1-z}
$$

and $q(z)=\frac{1+z}{1-z}$ is the best dominant.

Theorem 2.4. Let $q$ be univalent in $U$, with $q(0)=1$. Let $\sigma \in \mathbb{C}^{*}$ and $t, \nu, \eta \in \mathbb{C}$ with $\nu+\eta \neq 0$. Let $f \in \mathcal{A}$ and suppose that $f$ and $g$ satisfy the next conditions

$$
\frac{v I^{m+1}(f(z))+\eta I^{m+2}(f(z))}{(v+\eta) z} \neq 0, z \in U
$$

and

$$
\operatorname{Re}\left\{1+\frac{z q^{\prime \prime}(z)}{q^{\prime}(z)}\right\}>\max \{0,-\operatorname{Re} t\}, z \in U
$$

If

$$
\begin{aligned}
\psi(z) & =t\left[\frac{v I^{m+1}(f(z))+\eta I^{m+2}(f(z))}{(v+\eta) z}\right]^{\sigma} \\
& +\sigma\left[\frac{v z\left(I^{m+1}(f(z))\right)^{\prime}+z \eta\left(I^{m+2}(f(z))\right)^{\prime}}{v I^{m+1}(f(z))+\eta I^{m+2}(f(z))}-1\right]
\end{aligned}
$$

and

$$
\psi(z) \prec t q(z)+\frac{z q^{\prime}(z)}{q(z)}
$$

then

$$
\left[\frac{v I^{m+1}(f(z))+\eta I^{m+2}(f(z))}{(v+\eta) z}\right]^{\sigma} \prec q(z)
$$

and $q$ is the best dominant.

Proof. Let

$$
p(z)=\left[\frac{v I^{m+1}(f(z))+\eta I^{m+2}(f(z))}{(v+\eta) z}\right]^{\sigma}, z \in U .
$$

According to (2.3) the function $p(z)$ is analytic in $U$ and differentiating (2.10) logarithmically with respect to $z$, we obtain

$$
\frac{z p^{\prime}(z)}{p(z)}=\sigma\left[\frac{v z\left(I^{m+1}(f(z))\right)^{\prime}+z \eta\left(I^{m+2}(f(z))\right)^{\prime}}{v I^{m+1}(f(z))+\eta I^{m+2}(f(z))}-1\right]
$$

and hence

$z p^{\prime}(z)=\sigma\left[\frac{v I^{m+1}(f(z))+\eta I^{m+2}(f(z))}{(v+\eta) z}\right]^{\sigma} \cdot\left[\frac{v z\left(I^{m+1}(f(z))\right)^{\prime}+z \eta\left(I^{m+2}(f(z))\right)^{\prime}}{v I^{m+1}(f(z))+\eta I^{m+2}(f(z))}-1\right]$.

In order to prove our result we will use Lemma 1.2. In this lemma we consider

$$
\theta(w)=t w \text { and } \varphi(w)=\frac{1}{w}
$$


then $\theta$ is analytic in $\mathbb{C}$ and $\varphi(w) \neq 0$ is analytic in $\mathbb{C}^{*}$. Also if we let

$$
Q(z)=z q^{\prime}(z) \varphi(q(z))=\sigma\left[\frac{v z\left(I^{m+1}(f(z))\right)^{\prime}+z \eta\left(I^{m+2}(f(z))\right)^{\prime}}{v I^{m+1}(f(z))+\eta I^{m+2}(f(z))}-1\right]
$$

and

$$
\begin{gathered}
h(z)=\theta(q(z))+Q(z) \\
=t\left[\frac{v I^{m+1}(f(z))+\eta I^{m+2}(f(z))}{(v+\eta) z}\right]^{\sigma}+\sigma\left[\frac{v z\left(I^{m+1}(f(z))\right)^{\prime}+z \eta\left(I^{m+2}(f(z))\right)^{\prime}}{v I^{m+1}(f(z))+\eta I^{m+2}(f(z))}-1\right]
\end{gathered}
$$

from (2.6) we see that $Q(z)$ is a starlike function in $U$. We also have

$$
\operatorname{Re}\left\{\frac{z h^{\prime}(z)}{Q(z)}\right\}=\operatorname{Re}\left\{t+1+\frac{z q^{\prime \prime}(z)}{q^{\prime}(z)}\right\}>0 \text { for } z \in U
$$

and then, by using Lemma 1.2 we deduce that the subordination (2.9) implies $p(z) \prec q(z)$.

Taking $q(z)=\frac{1+A z}{1+B z}(-1 \leq B<A \leq 1)$ in Theorem 2.4 and according to

$$
\frac{z p^{\prime}(z)}{p(z)}=\sigma\left(\frac{I^{m+1}(f(z))}{I^{m+2}(f(z))}-1\right)
$$

the condition $(2.7)$ becomes $\max \{0,-\operatorname{Re}(t)\} \leq \frac{1-|B|}{1+|B|}$. Hence, for the special case $v=1$ and $\eta=0$ we obtain the following result.

Corollary 2.5. Let $t \in \mathbb{C}$ with $\max \{0,-\operatorname{Re}(t)\} \leq \frac{1-|B|}{1+|B|}$. Let $f \in \mathcal{A}$ and suppose that

$$
\frac{I^{m+1}(f(z))}{z} \neq 0, z \in U \text {. }
$$

If

$$
t\left[\frac{I^{m+1}(f(z))}{z}\right]^{\sigma}+\sigma\left[\frac{z\left(I^{m+1}(f(z))\right)^{\prime}}{I^{m+1}(f(z))}-1\right] \prec t \frac{1+A z}{1+B z}+\frac{(1-B) z}{(1+A z)(1+B z)}
$$

then

$$
\left(\frac{I^{m+1}(f(z))}{z}\right)^{\sigma} \prec \frac{1+A z}{1+B z}
$$

and $q(z)=\frac{1+A z}{1+B z}$ is the best dominant.

Taking $v=m=1, \eta=0$ and $q(z)=\frac{1+z}{1-z}$ in Theorem 2.1, we obtain the next result.

Corollary 2.6. Let $f \in \mathcal{A}$ and suppose that $\frac{I^{2}(f(z))}{z} \neq 0, z \in U, \sigma \in \mathbb{C}^{*}$. If

$$
t\left[\frac{I^{2}(f(z))}{z}\right]^{\sigma}+\sigma\left[\frac{z\left(I^{2}(f(z))\right)^{\prime}}{I^{2}(f(z))}-1\right] \prec t \frac{1+z}{1-z}+\frac{2 z}{(1+z)(1-z)}
$$

then

$$
\left[\frac{I^{2}(f(z))}{z}\right]^{\sigma} \prec \frac{1+z}{1-z}
$$

and $q(z)=\frac{1+z}{1-z}$ is the best dominant. 
Theorem 2.7. Let $q$ be convex in $U$, with $q(0)=1$. Let $\sigma \in \mathbb{C}^{*}$ and $t, \nu, \eta \in \mathbb{C}$ with $\nu+\eta \neq 0$ and $\operatorname{Re} t>0$. Let $f \in \mathcal{A}$ and suppose that $f$ satisfies the next conditions:

$$
\frac{v I^{m+1}(f(z))+\eta I^{m+2}(f(z))}{(v+\eta) z} \neq 0, z \in U
$$

and

$$
\left[\frac{v I^{m+1}(f(z))+\eta I^{m+2}(f(z))}{(v+\eta) z}\right]^{\sigma} \in \mathcal{H}[q(0), 1] \cap Q .
$$

If the function $\psi$ given by (2.8) is univalent in $U$ and

$$
t q(z)+\frac{z q^{\prime}(z)}{q(z)} \prec \psi(z),
$$

then

$$
q(z) \prec\left[\frac{v I^{m+1}(f(z))+\eta I^{m+2}(f(z))}{(v+\eta) z}\right]^{\sigma}
$$

and $q(z)$ is the best subordinant of (2.14).

Proof. Let

$$
p(z)=\left[\frac{v I^{m+1}(f(z))+\eta I^{m+2}(f(z))}{(v+\eta) z}\right]^{\sigma}, z \in U .
$$

According to (2.12) the function $p(z)$ is analytic in $U$ and differentiating (2.15) logarithmically with respect to $z$, we obtain

$$
\frac{z p^{\prime}(z)}{p(z)}=\sigma\left[\frac{v z\left(I^{m+1}(f(z))\right)^{\prime}+z \eta\left(I^{m+2}(f(z))\right)^{\prime}}{v I^{m+1}(f(z))+\eta I^{m+2}(f(z))}-1\right] .
$$

In order to prove our result we will use Lemma 1.4. In this lemma we consider

$$
Q(z)=z q^{\prime}(z) \varphi(q(z))=\sigma\left[\frac{v z\left(I^{m+1}(f(z))\right)^{\prime}+z \eta\left(I^{m+2}(f(z))\right)^{\prime}}{v I^{m+1}(f(z))+\eta I^{m+2}(f(z))}-1\right]
$$

and

$$
\begin{gathered}
h(z)=\theta(q(z))+Q(z) \\
=t\left[\frac{v I^{m+1}(f(z))+\eta I^{m+2}(f(z))}{(v+\eta) z}\right]^{\sigma}+\sigma\left[\frac{v z\left(I^{m+1}(f(z))\right)^{\prime}+z \eta\left(I^{m+2}(f(z))\right)^{\prime}}{v I^{m+1}(f(z))+\eta I^{m+2}(f(z))}-1\right]
\end{gathered}
$$

from (2.12) we see that $Q(z)$ is a starlike function in $U$. We also have

$$
\operatorname{Re}\left\{\frac{z h^{\prime}(z)}{Q(z)}\right\}=\operatorname{Re}\left\{t+1+\frac{z q^{\prime \prime}(z)}{q^{\prime}(z)}\right\}>0 \text { for } z \in U
$$

and then, by using Lemma 1.4 we deduce that the subordination (2.14) implies $q(z) \prec p(z)$ and the proof is completed. 
Corollary 2.8. Let $q_{1}, q_{2}$ are two convex functions in $U$, with $q_{1}(0)=q_{2}(0)=1$, $\sigma \in \mathbb{C}^{*}, t, \nu, \eta \in \mathbb{C}$ with $\nu+\eta \neq 0$ and $\operatorname{Ret}>0$. Let $f \in \mathcal{A}$ and suppose that $f$ satisfies the next conditions:

$$
\frac{v I^{m+1}(f(z))+\eta I^{m+2}(f(z))}{(v+\eta) z} \neq 0, z \in U
$$

and

$$
\left(\frac{v I^{m+1}(f(z))+\eta I^{m+2}(f(z))}{(v+\eta) z}\right)^{\sigma} \in \mathcal{H}[q(0), 1] \cap Q .
$$

If the function $\psi(z)$ given by $(2.8)$ is univalent in $U$ and

then

$$
t q_{1}(z)+\frac{z q_{1}^{\prime}(z)}{q_{1}(z)} \prec \psi(z) \prec t q_{2}(z)+\frac{z q_{2}^{\prime}(z)}{q_{2}(z)}
$$

$$
q_{1}(z) \prec\left(\frac{v I^{m+1}(f(z))+\eta I^{m+2}(f(z))}{(v+\eta) z}\right)^{\sigma} \prec q_{2}(z)
$$

and $q_{1}, q_{2}$ are respectively, the best subordinant and the best dominant of (2.17).

\section{References}

[1] Bulboacă, T., Classes of first-order differential superordinations, Demonstr. Math., 35(2002), no. 2, 287-292.

[2] Bulboacă, T., Differential Subordinations and Superordinations, Recent Results, House of Scientific Book Publ., Cluj-Napoca, 2005.

[3] Miller, S.S., Mocanu, P.T., Differential Subordinations: Theory and Applications, in Series on Monographs and Textbooks in Pure and Applied Mathematics, No. 225, Marcel Dekker, New York, 2000.

[4] Miller, S.S., Mocanu, P.T., Subordinants of differential superordinations, Complex Variables, 48(10)(2003), 815-826.

[5] Miller, S.S., Mocanu, P.T., Briot-Bouquet differential superordinations and sandwich theorems, J. Math., Anal. Appl., 329(2007), no. 1, 327-335.

[6] Sălăgean, G. Şt., Subclasses of univalent functions, Complex Analysis, Fifth RomanianFinnish Seminar, Part 1 (Bucharest, 1981), Lecture Notes in Math., vol. 1013, Springer, Berlin, 1983, 362-372.

Luminița-Ioana Cotîrlă

Technical University of Cluj-Napoca,

Department of Mathematics,

Cluj-Napoca, Romania

e-mail: luminita.cotirla@yahoo.com, Luminita.Cotirla@math.utcluj.ro

Adriana Cătaş

University of Oradea,

Department of Mathematics and Computer Science,

1, University Street, 410087 Oradea, Romania

e-mail: acatas@gmail.com 